\title{
Implementation of Lightweight Personalized Sensor Platform Based on Embedded
}

\author{
Hojoong Kim ${ }^{1}$, Wonhee Lee ${ }^{2}$, Jieun Jung ${ }^{3}$, Junho Shin ${ }^{4}$ and Byunghun Song* \\ Korea Electronics Technology Institute (KETI)

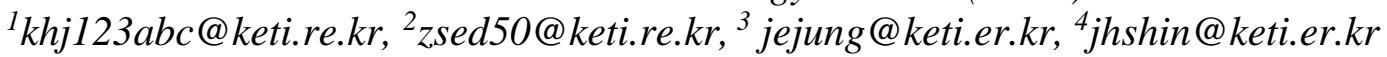 \\ "bhsong@keti.er.kr
}

\begin{abstract}
With the technological development of the manufacturing components and the IoT (Internet of Things), the demand for lightweight platform technology for personalized sensor information services has dramatically increased. In this paper, we propose a lightweight personalized sensor platform based on embedded system and present the implementation of sensor nodes, gateways, and sensor portals those are fundamental components for personalized sensor information services with OID (Object Identifier).
\end{abstract}

Keywords: Lightweight Sensor Node, IoT Platform, OID

\section{Introduction}

With the recent technological development of the manufacturing components and the IoT (Internet of Things), the environmental information service system can provide more information and support a lightweight platform[1-3]. And with the introduction of big data and cloud technologies, it is possible to analyze, process and inquire data collected from a remote site. As a result, the demand for lightweight platform technology to collect various environmental information and provide personalized environmental information services has dramatically increased.

In addition, with the development of sensor using MEMS (Micro Electro Mechanical Systems) technology, sensors became much more precise, smaller and cheaper. As a result, sensors with various functions and interface types are released, and the demand for highly compatible platform device has increased.

Therefore, in this paper, we proposed a lightweight personalized sensor platform based on embedded system collecting environmental information from various indoor/outdoor sensor modules, as well as designed and implemented the implementation of sensor nodes, gateways, and sensor portals those are fundamental components for personalized sensor information services with OID (Object Identifier) for the personalized environmental information service. And with operation of the implemented platform, we confirmed that it is possible to interlink environmental information of sensor nodes, gateways and sensor portals, and to provide a personalized environmental information service based on the interlinked environmental information.

\section{Lightweight Personalized Sensor Platform}

\subsection{Overall Architecture}

Lightweight personalized sensor platform based on embedded system is composed of sensor nodes, gateways and sensor portals. To lighten the platform, sensor nodes and gateways are based on the embedded system. To provide environmental information in

Received (June 10, 2017), Review Result (September 30, 2017), Accepted (October 16, 2017) 
real time and bi-directionally, gateways and sensor nodes carry out $1: \mathrm{N}$ wireless communication via Wi-Fi, and gateways carry out $\mathrm{N}: \mathrm{N}$ communication with sensor portals via LTE. Figure 1 show the structure of the personalized sensor platform based on embedded system.

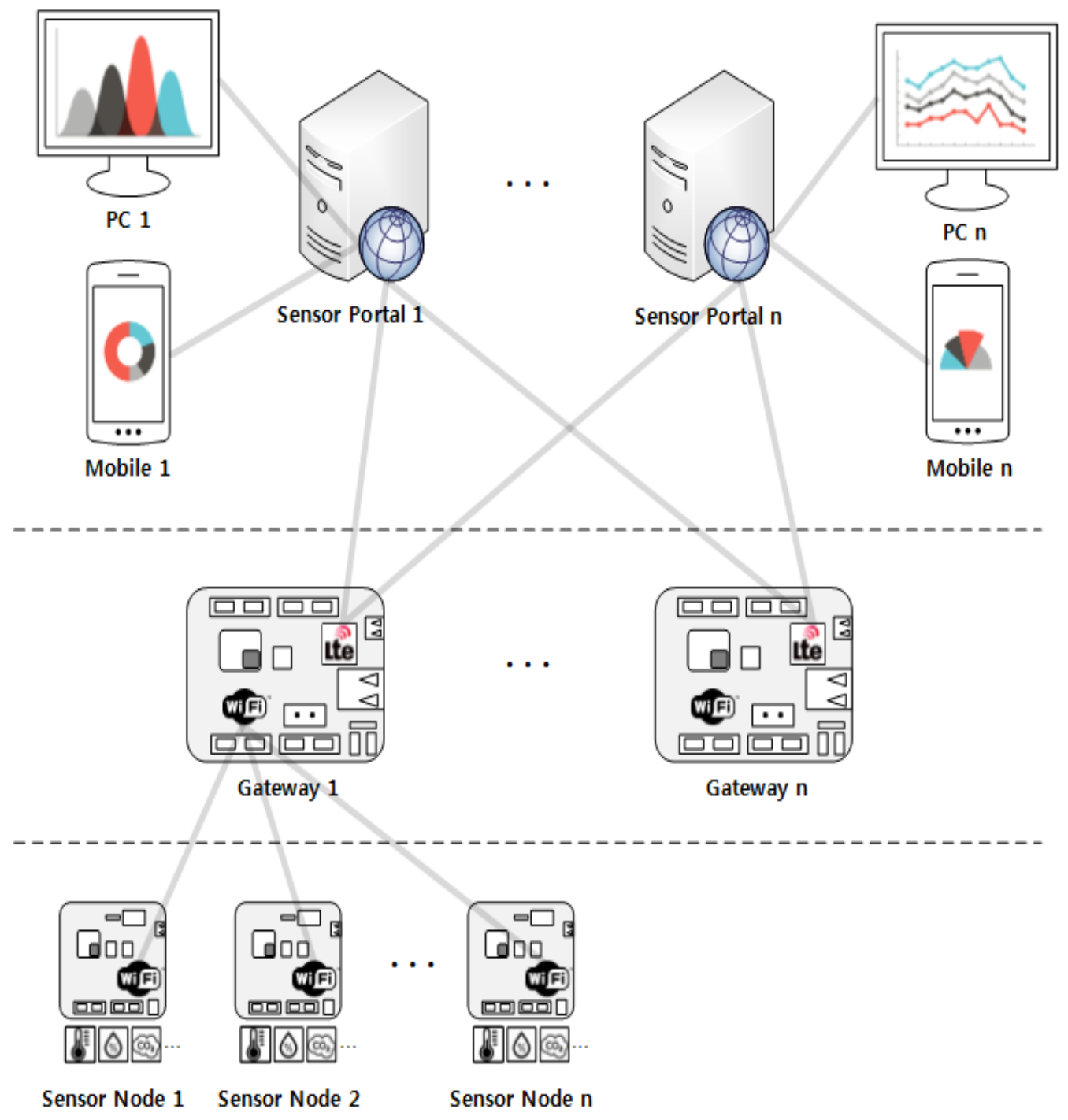

\section{Figure 1. Structure of the Lightweight Personalized Sensor Platform Based On Embedded System}

\subsection{Composition of Sensor Node}

As shown in Figure 2, sensor node is composed of an interface device and an end device that detects environmental information. It is connected to various environmental information measurement sensors, and installed in proper places to collect environment measurement information. Sensor node sends the collected information to nearby gateways and platform servers on a regular basis. And depending on the situation, it inquires the environmental information and controls the interlinked device in real time.

Interface device of sensor node collects data from the end device that defects the environmental information. Then it processes the information and sends it to the gateway. End device is composed of many products including sensor module using UART, I2C, Digital or Analog as its interface type, commercial device in the market and the selfdeveloped sensor device. It detects the environmental information. 


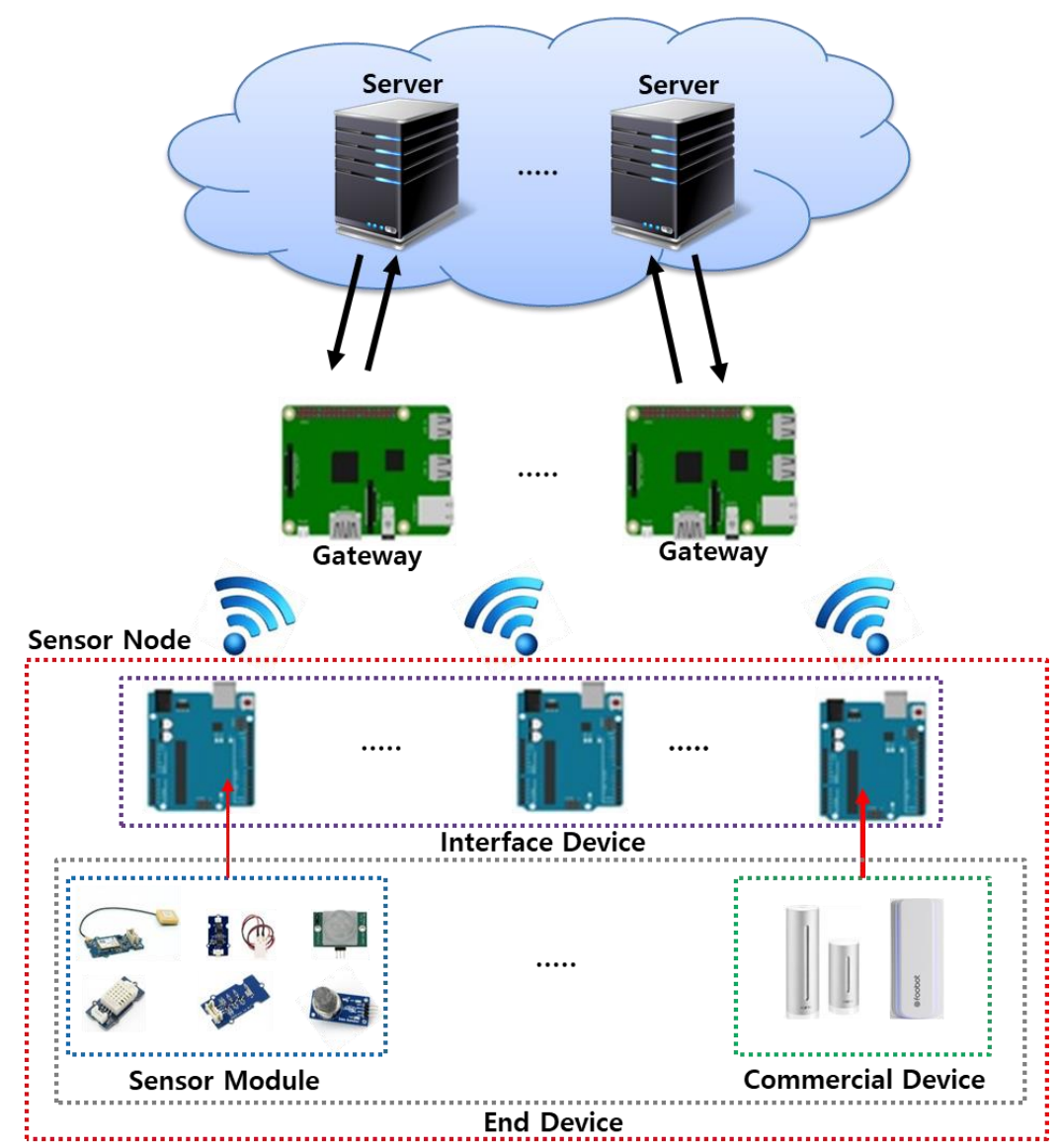

Figure 2. Composition of Sensor Node

Figure 3 (a) shows the sensor module used in this study. It detects the environmental information using Gas, GPS, vibration, temperature/humidity, movement, air pressure, UV rays, illumination intensity and camera modules[4]. In addition, to improve compatibility and scalability of sensor platform, we collected the environmental information detected by commercial devices including Foobot[5], Netamo[6] and Kweather[7]. Figure 3 (b) shows the commercial devices applied in this study.
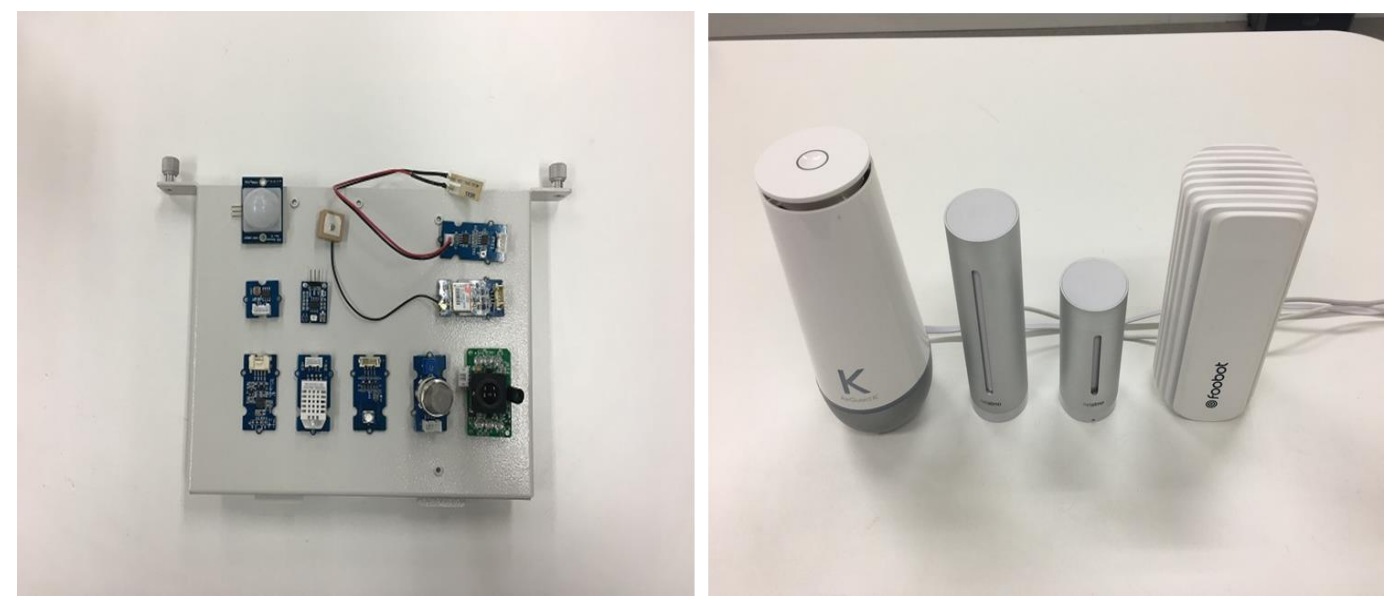

Figure 3. (a) Sensor Module, (b) Commercial Devices to Collect the Environmental Information 
Figure 4 (a) shows the interface device that collects the environmental information detected by the sensor module and commercial devices. Interface device is a microcontroller based on ATmega2560[8]. It is connected to the sensor module and commercial devices in many different types to collect data. In this study, we secured an independent interface device by designing a PCB and built the final sensor node, as shown in Figure 4 (b)

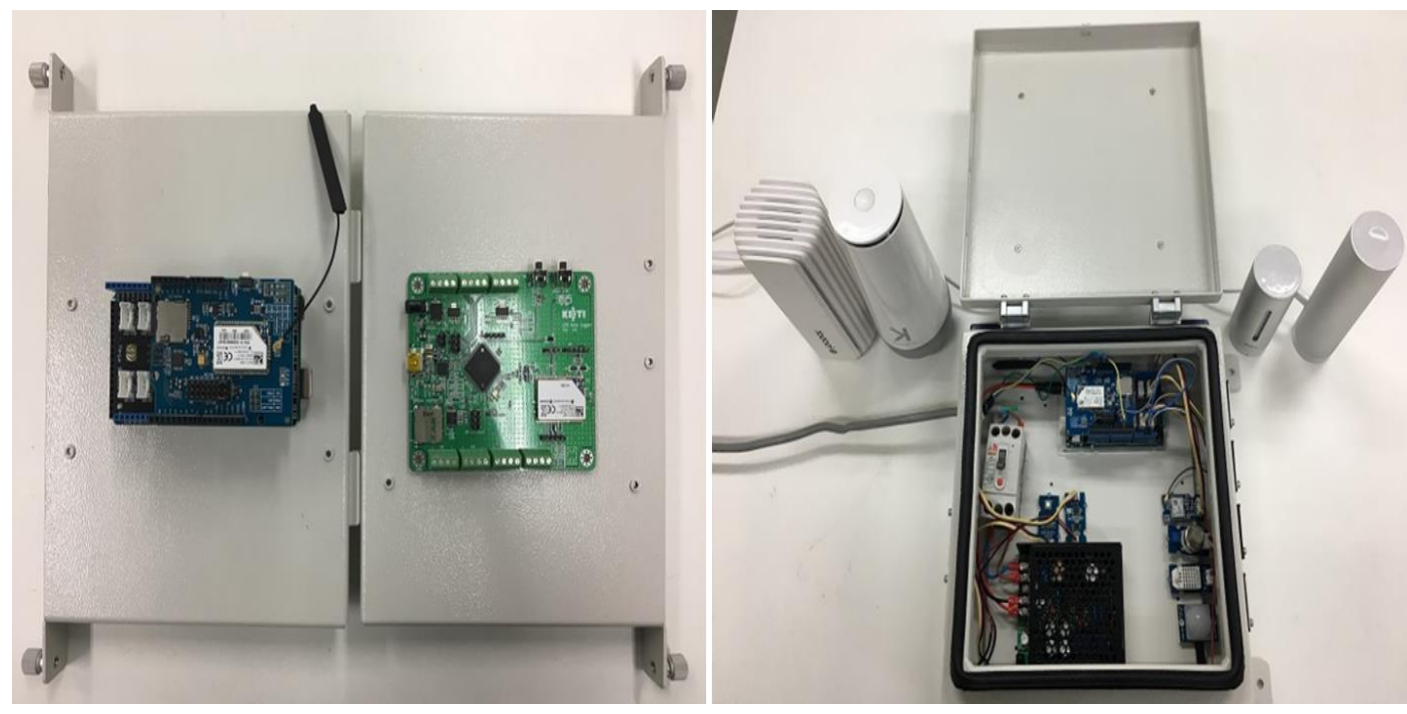

Figure 4. (a) Interface Device, (b) the Final Sensor Node that Collects the Environmental Information Defected by Sensor Module and Commercial Devices

Figure 5 (a) is the transmission of the information, collected by connecting 7 sensor modules and 1 commercial device with interface device, to the gateway. Figure 5 (b) is the result of the gateway's reception of environmental information, collected by the sensor platform, via WI-FI. We confirmed that this sensor node we built can be operated as a highly compatible device, and as a base equipment to provide various services in various fields.
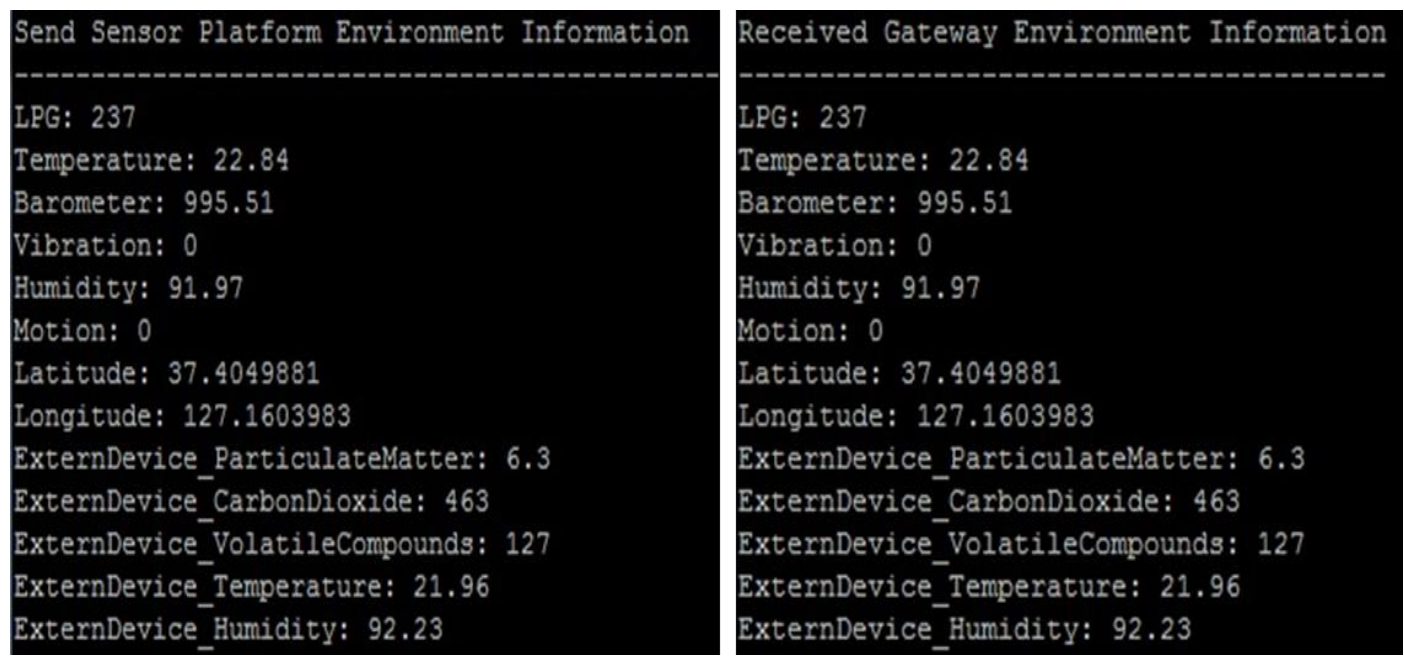

Figure 5. (a) Sensor Node Transmission Log, (b) Gateway Reception Log

\subsection{Composition of Gateway and Portal}


Gateway collects information of various sensor nodes, carries out the data integration and protocol conversion, in order to send the environmental information to sensor portal. Also, it sends orders to inquire the environmental information requested by sensor portal and to control the interlinked device to sensor node. Gateway is composed based on low power ARM-based processor, and it was possible to confirm that the environmental information was integrated and sent to the sensor portal with a converted protocol. Finally, the sensor portal classifies the environmental information sent to the gateway based on OID (Object Identifier), and provides personalized environmental information services with a user-friendly UI. In addition, with mobile synchronization, user can check the environmental information in real time.

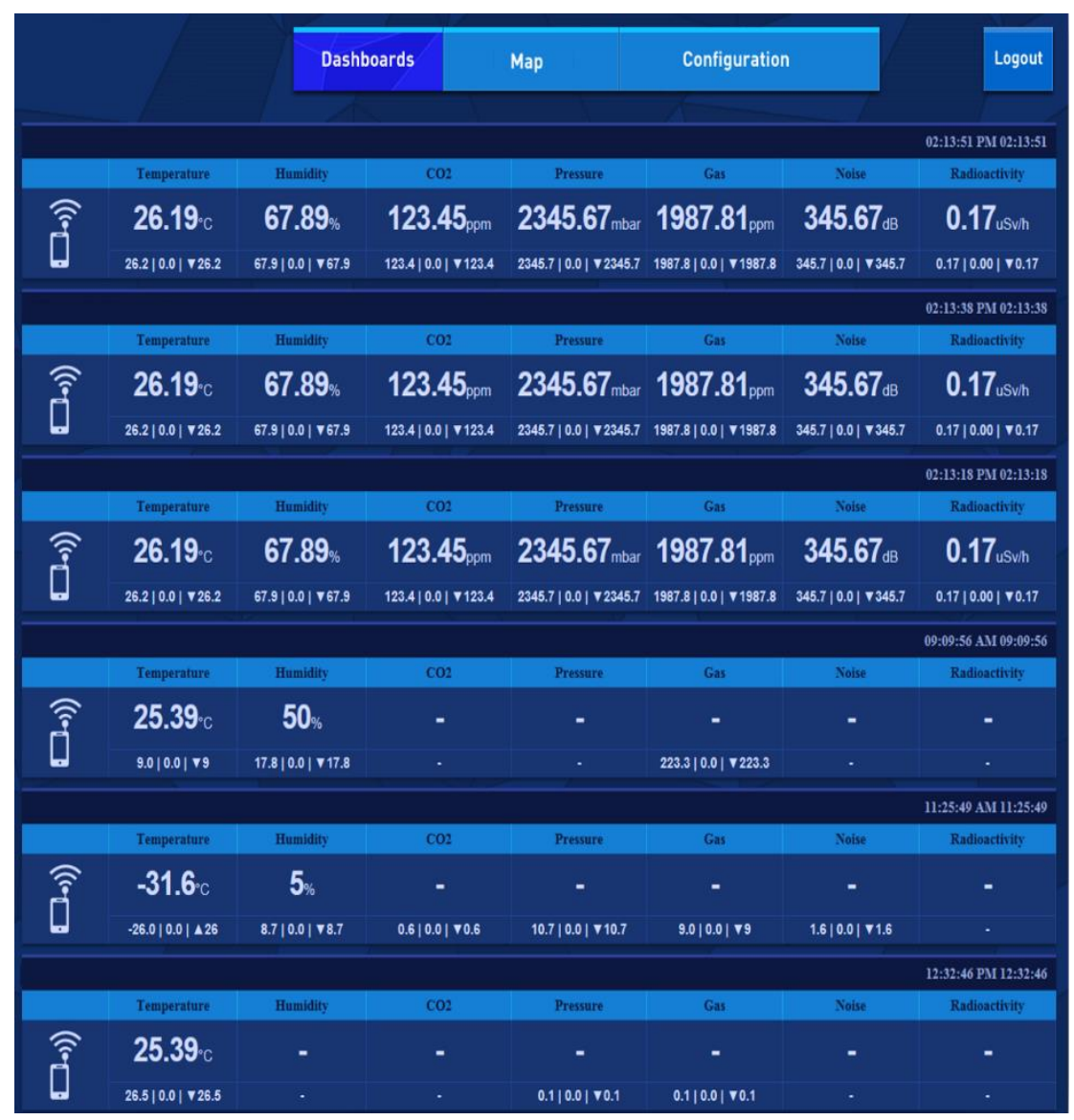

Figure 6. Sensor Portal Screen

\subsection{Composition of OID}

OID for the personalized environmental information service is a unique identification system used to control and maintain each device's information from the lightweight personalized sensor platform based on embedded system. Table 1 shows the OID for the personalized environmental information service. It is composed of information including nation, registration organization, project, related organization, device and serial number. All information between sensor node, gateway and sensor portal is sent with OID, and it is possible to provide users with personalized environmental information services by identifying information and differentiating users with OID. 
Table 1. OID for Personalization and Environmental Information Service

\begin{tabular}{|c|c|c|c|c|c|c|c|c|c|c|c|c|}
\hline \multicolumn{11}{|c|}{ OID } & \multirow{2}{*}{ 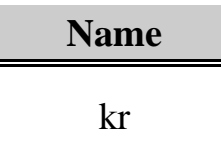 } & \multirow{2}{*}{$\begin{array}{l}\text { Meaning } \\
\text { Republic } \\
\text { of Korea }\end{array}$} \\
\hline 1.2 .410 & & & & & & & & & & & & \\
\hline 1.2 .410 & 2000060 & & & & & & & & & & keti & KETI \\
\hline 1.2 .410 & 2000060 & 1 & & & & & & & & & $\begin{array}{l}\text { sensor- } \\
\text { platform }\end{array}$ & $\begin{array}{c}\text { Lightweight } \\
\text { Personalized } \\
\text { Sensor } \\
\text { Platform } \\
\text { Based on } \\
\text { Embedded } \\
\text { System } \\
\end{array}$ \\
\hline 1.2 .410 & 2000060 & 1 & 1 & & & & & & & & keti & KETI \\
\hline 1.2 .410 & 2000060 & 1 & 1 & 1 & & & & & & & potal & Gateway \\
\hline 1.2 .410 & 2000060 & 1 & 1 & 1 & 1 & & & & & & serial-1 & $\begin{array}{c}\text { Serial } \\
\text { Number } 1\end{array}$ \\
\hline \multicolumn{13}{|c|}{ etc } \\
\hline 1.2 .410 & 2000060 & 1 & 1 & 1 & 1 & 1 & & & & & gateway & $\begin{array}{l}\text { Sensor } \\
\text { Node }\end{array}$ \\
\hline 1.2 .410 & 2000060 & 1 & 1 & 1 & 1 & 1 & 1 & & & & serial-1 & $\begin{array}{c}\text { Serial } \\
\text { Number } 1\end{array}$ \\
\hline \multicolumn{13}{|c|}{ etc } \\
\hline 1.2 .410 & 2000060 & 1 & 1 & 1 & 1 & 1 & 1 & 1 & & & sensor & Sensor \\
\hline 1.2 .410 & 2000060 & 1 & 1 & 1 & 1 & 1 & 1 & 1 & 1 & & $\begin{array}{l}\text { temperature- } \\
\text { sensor }\end{array}$ & $\begin{array}{c}\text { Temperature } \\
\text { sensor }\end{array}$ \\
\hline 1.2 .410 & 2000060 & 1 & 1 & 1 & 1 & 1 & 1 & 1 & 1 & 1 & serial-1 & $\begin{array}{c}\text { Serial } \\
\text { Number } 1\end{array}$ \\
\hline 1.2 .410 & 2000060 & 1 & 1 & 1 & 1 & 1 & 1 & 1 & 2 & & $\begin{array}{l}\text { humidity- } \\
\text { sensor }\end{array}$ & $\begin{array}{l}\text { Humidity } \\
\text { sensor }\end{array}$ \\
\hline
\end{tabular}

\section{Conclusion}

This article proposed a lightweight personalized sensor platform based on embedded system, and composed an embedded system with sensor nodes and gateways and operated it. As a result, we confirmed that it is possible to provide personalized environmental information services by interlinking with sensor portal. It is considered that more studies are required to collect data obtain from the lightweight personalized sensor platform based on embedded system, make it as a big data, then provide it for a specific group or users in a specific area as a public information. In addition, it is required to conduct studies based on the broadening of IoT device being conducted by telecommunication companies. The purpose if this study is to build a highly compatible sensor platform for many devices to detect the environmental information. It was possible to collect the environmental information using various sensor modules for industrial/domestic uses and commercial devices, and utilize the collected environmental information as various types of service by sending it securely to the upper level gateway. In future, we'll conduct studies on the expansion of the platform with other wireless communication technologies except WI-FI, and studies using image modules. 


\section{Acknowledgments}

This work was supported by "Development of Open Industry IoT (IIoT) Smart Factory Platform and Factory-Thing Hardware Technology" of Korea Evaluation Institute of Industrial Technology (KEIT) granted financial resource from the Ministry of Trade, Industry \& Energy, Republic of Korea (No. 10054486)

This work was supported by the International Collaborative Energy Technology R\&D Program of the Korea Institute of Energy Technology Evaluation and Planning (KETEP) granted financial resource from the Ministry of Trade, Industry \& Energy, Republic of Korea (No. 20148530050120)

\section{References}

[1] K. Martinez, J. K. Hart and R. Ong, "Environmental sensor networks", IEEE Computer Society, (2004), pp. 50-56.

[2] A. Mainwaring, D. Culler, J. Polastre, R. Szewczyk and J. Anderson, "Wireless sensor networks for habitat monitoring", ACM International Workshop on Wireless Sensor Networks and Applications, (2002), pp. 88-97.

[3] R. Cardell-Oliver, K. Smettem, M. Kranz and K. Mayer, "A reactive soil moisture sensor network: Design and field evaluation”, International Journal of Distributed Sensor Networks, vol. 1, no. 2, (2005), pp. 149-162.

[4] Seeed Wiki, http://wiki.seeed.cc/Sensor [Accessed: August. 07, 2017].

[5] Foobot, http://foobot.io [Accessed: August. 07, 2017].

[6] Netatmo, https://www.netatmo.com [Accessed: August. 07, 2017].

[7] Kweather, http://www.kweather.co.kr/ [Accessed: August. 07, 2017]

[8] ATmega2560, http://www.atmel.com [Accessed: August. 07, 2017].

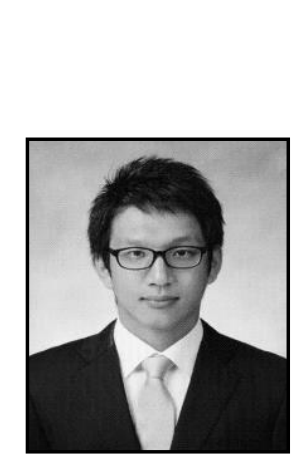

\section{Authors}

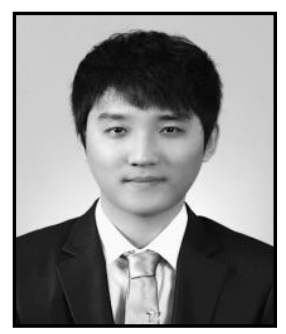

Hojoong Kim, he is a Researcher in IoT Platform Research Center at Korea Electronics Technology Institute (KETI). He received BS and MS in electronic engineering from Inha university, South Korea, in 2011 and 2015 respectively. His research interests are in the areas of IoT and Embedded System.

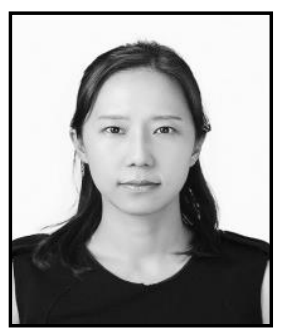

Jieun Jung, she is a senior researcher in IoT Platform Research Center at Korea Electronics Technology Institute (KETI). She received her BS in computer engineering from Sogang university, and MS in computer science from KAIST, South Korea in 2007 and 2009, respectively Her research interests and activities are in areas of Industrial IoT platform, process data mining, and standard for M2M/IoT services. 


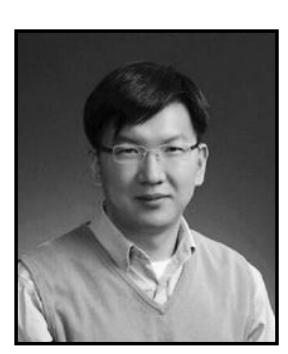

Junho Shin, he is a Managerial Researcher in IoT Platform Research Center at Korea Electronics Technology Institute (KETI). $\mathrm{He}$ received $\mathrm{BS}$ and $\mathrm{MS}$ in electronic engineering from Ajou university, South Korea, in 1998 and 2000 respectively. His research interests are in the areas of IoT, Sensor Network, and Embedded System.

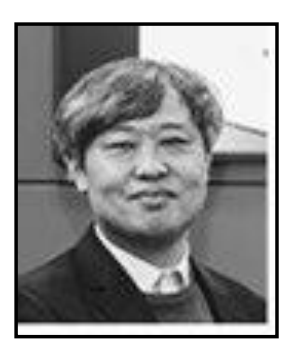

Byunghun Song, he is a head of Smart Factory ICT R\&D Center at Korea Electronics Technology Institute (KETI), Seongnam-si, South Korea. He received BS, MS and PhD degrees in electrical and electronic engineering from Kwangwoon university, South Korea, in 1998, 2000, and 2004 respectively. He serves as a leader of Infrastructure Team in Korea Smart Factory Foundation (KOSF) and a representative of OPC Foundation Korea. He has over 20 years of experience in the field of wireless sensor network and internet of things. He is now working in the Industrial Internet research center at KETI. His research interests and activities are in areas of Industrial IoT platform and infrastructure architecture for smart factory. 